\title{
Topology of Trace Deformed Yang-Mills Theory
}

\section{Marco Cardinali*}

University of Pisa and INFN-Pisa

E-mail: marco.cardinali@pi.infn.it

\section{Claudio Bonati}

University of Pisa and INFN-Pisa

E-mail: claudio.bonati@unipi.it

\section{Massimo D’Elia}

University of Pisa and INFN-Pisa

E-mail: massimo.delia@unipi.it

\section{Fabrizio Mazziotti}

University of Pisa and INFN-Pisa

E-mail: fabrizio.mazziotti@pi.infn.it

In this paper we study, by means of numerical simulations, the topological properties of $S U(3)$ and $S U$ (4) trace deformed Yang-Mills theory defined on $\mathbb{R}^{3} \times S^{1}$, in which center symmetry is recovered even at small compactification radii. In particular, we compute the topological suscpetibility $\chi$ and the coefficient $b_{2}$ (related to the fourth cumulant of the topological charge distribution). We find that these observables computed in the deformed theory when center symmetry is recovered are compatible with their values at zero temperature both for 3 and 4 colours.

37th International Symposium on Lattice Field Theory - Lattice2019

16-22 June 2019

Wuhan, China

\footnotetext{
* Speaker.
} 


\section{Introduction}

Pure gauge Yang-Mills theories, defined on a space-time with one or more compactified directions and periodic boundary conditions, are invariant under center symmetry transformations. A center symmetry transformation consists of a gauge transformation which respects the periodicity but for a global element of the center of the gauge group, in particular the center of $S U(N)$ is $\mathbb{Z}_{N}$. In the limit of small compactification radius, the system undergoes a phase transition and center symmetry is spontaneously broken; the order parameter of this phase transition is the holonomy around the compactified direction and it is called Polyakov loop. If we identify the compactified direction with the thermal Euclidean direction the spontaneous symmetry breaking of center symmetry is the well known deconfinement phase transition and the Polyakov loop is defined as

$$
P(\vec{x})=\mathscr{P} \exp \left(i \int_{0}^{L} A_{0}(\vec{x}, \tau) \mathrm{d} \tau\right)
$$

In the low- $T$ confined phase the trace of the Polyakov loop is zero, while it becomes non-zero in the high- $T$ (or small compactification length $L$ ) regime. The low- $T$ phase possesses several interesting properties such as confinement or the $\theta$ dependence; however, this regime is characterized by strong coupling and a perturbative description is not reliable. On the contrary, the high- $T$ regime is weakly coupled and it is possible, for example, to use semiclassical approximations to make predictions. Since the low- $T$ and high- $T$ phases are separeted by a phase transition they are not analitically connected and it is not possible to use the semiclassical methods mentioned before to describe the confining phase.

Trace deformed Yang-Mills was first studied on the lattice in [1] and then proposed as a possible way to overcome the problem of the phase transition in [2]. The deformation consists of extra pieces added to the usual Yang-Mills action which preserve center symmetry even at high- $T$. The discretized action of the theory for $S U(3)$ is the following:

$$
S^{\mathrm{def}}=S_{Y M}+h \sum_{\vec{n}}|\operatorname{Tr} P(\vec{n})|^{2}
$$

where $h$ is the coupling of the trace deformation and $S_{Y M}$ is the usual Wilson action. If the parameter $h$ is high enough, the extra piece suppresses gauge configurations in which the Polyakov loop acquires a non zero value; thus the system recover center symmetry even at high temperature.

In this paper we compute on the lattice two observables related to the topological properties of the deformed theory when center symmetry is recovered: the topological susceptibility $\chi$ and the coefficient $b_{2}$, which is related to the fourth cumulant of the topological charge distribution. A first study (see Ref. [3]) showed that, for $N=3$, both the topological susceptibility and $b_{2}$ reach a plateau value increasing the parameter $h$, and such values are compatible with the zero temperature ones. Inspired by this result we decided to extend the analysis to $N=4$ (see Ref. [4]), since in this case we have a less trivial center symmetry breaking pattern. In section 2 we will give a brief review of $\theta$ dependence and how to determine it on the lattice. Section 3 is dedicated to $S U(3)$ results, while section 4 to $S U(4)$. In the end we will summarize the main results in the conclusions. 


\section{2. $\theta$ Dependence and Lattice Setup}

The dependence on the topological $\theta$-parameter enters in the (Euclidean) Lagrangian as follows:

$$
\mathscr{L}_{\theta}=\frac{1}{4} F_{\mu v}^{a}(x) F_{\mu v}^{a}(x)-i \theta q(x),
$$

where $q(x)$ is the topological charge defined by

$$
q(x)=\frac{g^{2}}{64 \pi^{2}} \varepsilon_{\mu v \rho \sigma} F_{\mu v}^{a}(x) F_{\rho \sigma}^{a}(x) .
$$

The relevant information about $\theta$ dependence is contained in the free energy density, which around $\theta=0$ can be parametrized as follows [5]:

$$
f(\theta)=f(0)+\frac{1}{2} \chi \theta^{2}\left(1+b_{2} \theta^{2}+b_{4} \theta^{4}+\cdots\right)
$$

where the topological suscpetibility $\chi$ and all the coefficients $b_{2 n}$ can be related to the cumulants of the topological charge distribution computed at $\theta=0$ by the relations:

$$
\chi=\frac{\left\langle Q^{2}\right\rangle_{c, \theta=0}}{\mathscr{V}}, \quad b_{2 n}=(-1)^{n} \frac{2\left\langle Q^{2 n+2}\right\rangle_{c, \theta=0}}{(2 n+2) !\left\langle Q^{2}\right\rangle_{c, \theta=0}}
$$

in which $Q$ is the winding number defined as $Q=\int d^{4} x q(x)$ and $\mathscr{V}$ is the four-dimensional volume. Topological susceptibility is predicted to stay finite in the large- $N$ limit $[6,7,8]$ and in the low temperature, confined phase, while the other coefficients $b_{2 n}$ are suppressed as follows:

$$
\chi=\chi_{\infty}+O\left(N^{-2}\right), \quad b_{2 j}=O\left(N^{-2 j}\right) .
$$

Lattice simulations support such predictions, see Refs. $[9,12,10,11]$ for the topological susceptibility and Refs. [12, 13, 14, 15, 16, 17, 18] for the coefficient $b_{2}$.

On the contrary, in the high temperature deconfined regime the theory is weakly coupled and one expects that semiclassical arguments, such as instanton calculus, can be used to describe topology. In particular, the dilute instanton gas approximation (DIGA) leads to the following expressions:

$$
\begin{aligned}
f(\theta)-f(0) & \simeq \chi(T)(1-\cos \theta) \\
\chi(T) & \simeq T^{4} \exp \left[-8 \pi^{2} / g^{2}(T)\right] \sim T^{-\frac{11}{3} N+4},
\end{aligned}
$$

which predicts, in the large- $N$ limit, a constant value for the coefficients $b_{2 n}$ and a vanishing value for the topological susceptibility. Lattice simulations [10, 17, 19, 20, 21] performed above the deconfinement phase transistion are in agreement with the DIGA predictions; moreover, the transition between the low- $T$ and high- $T$ behaviour of the topology is sharper and sharper as $N$ increases.

In this paper we measure both $\chi$ and $b_{2}$ in the deformed theory for $S U(3)$ and $S U(4)$. We performed simulations using the deformed action (Wilson action plus deformations) at $\theta=0$ and then we computed $\chi$ on the gauge configurations using the standard gluonic definition, while the coefficient $b_{2}$ has been measured using the imaginary $\theta$ method exposed in [17]. 


\section{3. $S U(3)$ Results}

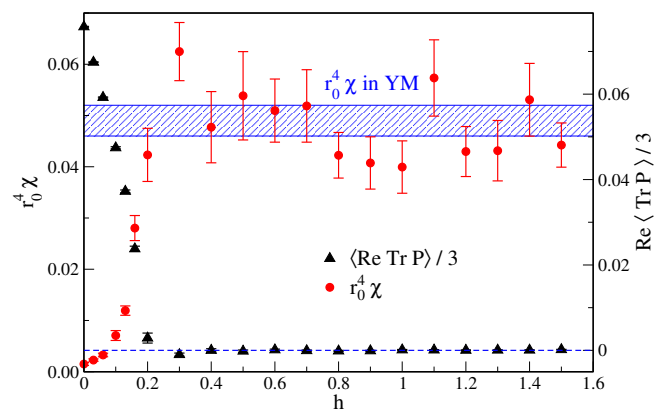

Figure 1: Topological susceptibility $\chi$ and the Polyakov loop $\operatorname{Re}\langle\operatorname{Tr} P\rangle / 3$ as functions of the parameter $h$. The lattice is $8 \times 32^{3}$ and the bare coupling is $\beta=6.4$. We also report for reference the standart $S U(3)$ value at zero temperature [5].

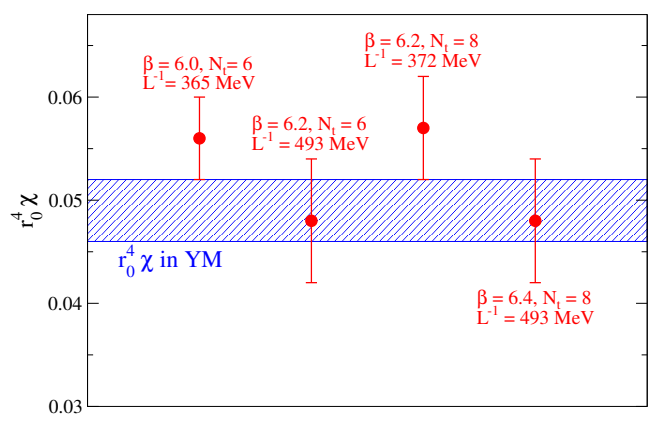

Figure 2: The plaetau value of the topological susceptibility for different temporal extensions and bare couplings. We also report for reference the standart $S U(3)$ value at zero temperature [5].

In Fig. 1 the topological susceptibility is shown as a function of $h$. The system is deep in the deconfined phase and when the deformation is off $(h=0) \chi$ is very small. As $h$ increases the topological susceptibility starts growing and reaches a plateau. The plateau is reached when $\operatorname{Re}\langle\operatorname{Tr} P\rangle / 3$ becomes zero, thus when center symmetry is recovered; the remarkable fact is that the plateau value is in agreement with the zero temperature one. In order to fix the scale we used the Sommer parameter $r_{0}$ fixing it at $r_{0}=0.5$ (see Ref. [23]) and we assumed that the deformation does not modify the lattice spacing. This has been checked explicitly in Ref. [3]. We performed the same measures also with different bare couplings and temporal extensions and the results are shown in Fig. 2; in all the cases the plateau values are in agreement with the zero temperature result. We show in Fig. 3 the values of the coefficient $b_{2}$ in the deformed theory for two values of $h$ in the plateau region. Even in this case the results are compatible with the zero temperature case, while they are different from the semiclassical high- $T$ predictions.

We also measured the topological susceptibility using higher values of the coupling $h$ and different lattice setups in order to check if there are any cut-off effects. We consider lattices with approximately the same temperature, but with finer lattice spacings. Results are shown in Fig. 4. From the figure we can understand that the plateau region is more stable when the lattice spacing is finer, i.e. when the continuum limit is approached. In particular we can see that the plateau region is not clearly defined for small $N_{t}$; a fact that suggests that the deformation may lead to severe cut-off effects when the temporal extension is too small.

We also checked possible finite-volume effects; we measured the topological susceptibility using a $8 \times 64^{3}$ lattice at $h=2.0, \beta=6.4$ and we compared it with the same value obtained on a $8 \times 32^{3}$ lattice. As we can see from Fig. 4, there are no visibile finite-volume effects.

\section{4. $S U(4)$ Results}

$S U$ (4) Yang-Mills theory has two possible ways to break center symmetry; either the symmetry is fully broken $\left(\mathbb{Z}_{4} \rightarrow \mathbb{1}\right)$ or there is a residual symmetry group left unbroken $\left(\mathbb{Z}_{4} \rightarrow \mathbb{Z}_{2}\right)$. 


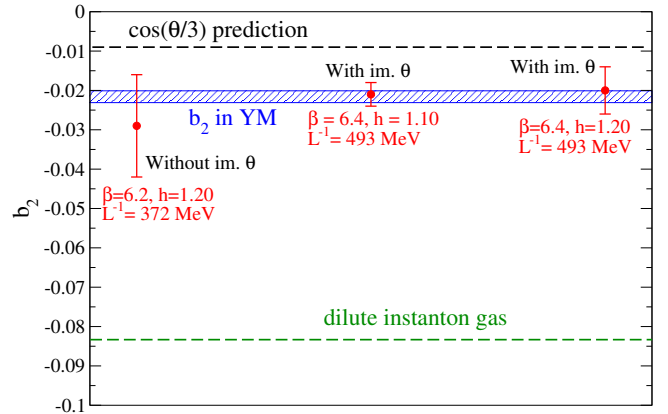

Figure 3: The coefficient $b_{2}$ measured using $8 \times 32^{3}$ lattices. The standard $S U(3)$ value [17] is the horizontal band, dashed lines represent the value $b_{2}=$ $-1 / 12$ (DIGA) and $b_{2}=-1 / 108$ (Fractional Instanton Gas Approximation [24, 25, 26]).

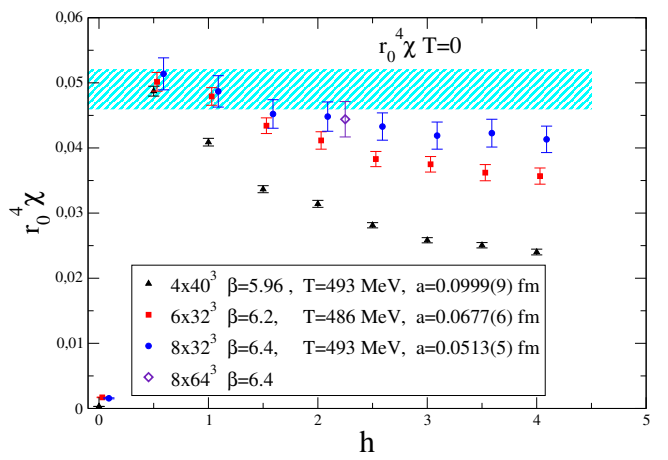

Figure 4: Topological susceptibility $\chi$ computed using different values of the coupling $h$ and using different lattice setups. The lattice spacing has been computed using $r_{0}=0.5$.

Therefore, in order to recover the full center symmetry we need two deformations: one proportional to $|\operatorname{Tr} P|$ and one proportional to $\left|\operatorname{Tr} P^{2}\right|$, where the second deformation is used to avoid the breaking to $\mathbb{Z}_{2}$ since the order parameter of this phase transition is $\operatorname{Tr} P^{2}$. The action used in the simulation is the following:

$$
S^{\mathrm{def}}=S_{Y M}+h_{1} \sum_{\vec{n}}|\operatorname{Tr} P(\vec{n})|^{2}+h_{2} \sum_{\vec{n}}\left|\operatorname{Tr} P^{2}(\vec{n})\right|^{2} .
$$

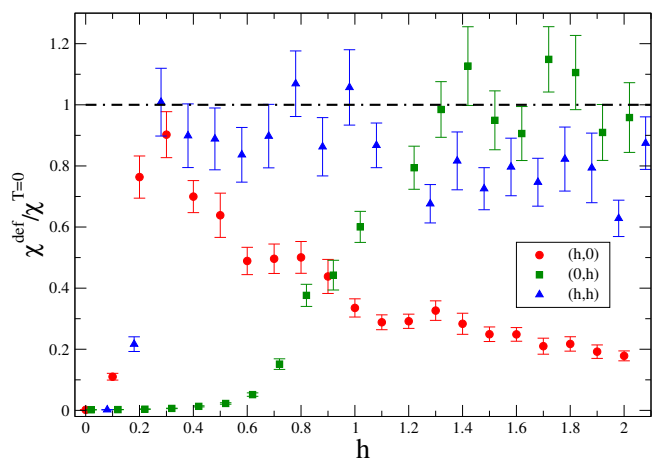

Figure 5: Topological susceptibility $\chi$ as a function of the parameters $h_{1}$ and $h_{2}$. The lattice is $6 \times 32^{3}$ and the bare coupling is $\beta=11.40$.

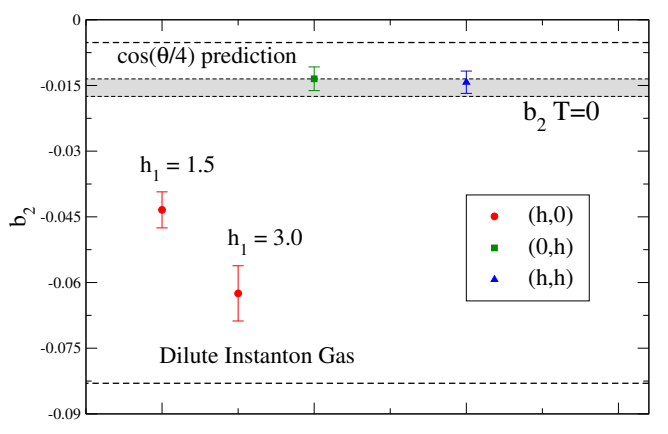

Figure 6: coefficient $b_{2}$ measured in the deformed theory on a $6 \times 32^{3}$ lattice at $\beta=11.40$ using one or both deformations; $h_{1}$ and $h_{2}$ have been chosen in the plateau region of the topological susceptibility.

In Figs. 5 and 6 we show the results for $S U$ (4). If we use both deformations (triangular points) the topological susceptibility and $b_{2}$ become compatible with the $T=0$ values while if we use only one of the deformation the situation is different. The term proportional to $|\operatorname{Tr} P|$ is not able to recover full center symmetry and both the topological suscpetibility and $b_{2}$ do not reach a plateau (circular points). However, if we use only the term proportional to $\left|\operatorname{Tr} P^{2}\right|$ we are able to recover full center symmetry and $\chi$ and $b_{2}$ behave as in the zero temperature case. 


\section{Conclusions}

We studied the $\theta$-dependence of the trace deformed Yang-Mills theory using 3 and 4 colors. The trace deformation consists of additional terms in the standard Yang-Mills action used to prevent the spontaneous symmetry breaking of center symmetry even at small compactification radii. We computed, by means of numerical simulations, the topological susceptibility $\chi$ and the coefficient $b_{2}$ related to the fourth order cumulant of the expansion of the topological charge distribution. Both in $S U(3)$ and $S U(4)$ these observables computed in the deformed theory are compatible with their values obtained at zero temperature; however, for $S U(4)$ we observed that center symmetry must be fully recovered in order to obtain the $T=0$ results.

\section{References}

[1] J. C. Myers and M. C. Ogilvie, New phases of $S U(3)$ and $S U(4)$ at finite temperature, Phys. Rev. D 77, 125030 (2008) [arXiv:0707.1869 [hep-lat]].

[2] M. Unsal and L. G. Yaffe, Center-stabilized Yang-Mills theory: Confinement and large N volume independence, Phys. Rev. D 78, 065035 (2008) [arXiv:0803.0344 [hep-th]].

[3] C. Bonati, M. Cardinali and M. D'Elia, $\theta$ dependence in trace deformed SU (3) Yang-Mills theory: a lattice study, Phys. Rev. D 98, no. 5, 054508 (2018) [arXiv:1807.06558 [hep-lat]].

[4] C. Bonati, M. Cardinali, M. D'Elia and F. Mazziotti, $\theta$ dependence and center symmetry in Yang-Mills theory, [arXiv:1912.02662 [hep-lat]].

[5] E. Vicari and H. Panagopoulos, Theta dependence of $S U(N)$ gauge theories in the presence of a topological term, Phys. Rept. 470, 93 (2009) [arXiv:0803.1593 [hep-th]].

[6] G. 't Hooft, A planar diagram theory for strong interactions, Nucl. Phys. B 72, 461 (1974).

[7] E. Witten, Large N Chiral Dynamics, Annals Phys. 128, 363 (1980).

[8] E. Witten, Theta dependence in the large-N limit of four-dimensional gauge theories, Phys. Rev. Lett. 81, 2862 (1998).

[9] B. Lucini and M. Teper, $S U(N)$ gauge theories in four-dimensions: Exploring the approach to $N=$ infinity, JHEP 0106, 050 (2001) [hep-lat/0103027].

[10] B. Lucini, M. Teper, U. Wenger, Topology of $S U(N)$ gauge theories at $T=O$ and $T=T(c)$, Nucl. Phys. $B$ 715, 461 (2005) arXiv:hep-lat/0401028.

[11] M. Cè, M. Garcìa Vera, L. Giusti and S. Schaefer, The topological susceptibility in the large-N limit of SU(N) Yang Mills theory, Phys. Lett. B 762, 232 (2016) [arXiv:1607.05939 [hep-lat]].

[12] L. Del Debbio, H. Panagopoulos and E. Vicari, theta dependence of $S U(N)$ gauge theories, JHEP 0208, 044 (2002) [hep-th/0204125].

[13] M. D'Elia, Field theoretical approach to the study of theta dependence in Yang-Mills theories on the lattice, Nucl. Phys. B 661, 139 (2003) [hep-lat/0302007].

[14] L. Giusti, S. Petrarca and B. Taglienti, Theta dependence of the vacuum energy in the SU(3) gauge theory from the lattice, Phys. Rev. D 76, 094510 (2007) [arXiv:0705.2352 [hep-th]].

[15] H. Panagopoulos and E. Vicari, The $4 D S U(3)$ gauge theory with an imaginary $\theta$ term, JHEP 1111, 119 (2011) [arXiv:1109.6815 [hep-lat]]. 
[16] M. Cè, C. Consonni, G. P. Engel and L. Giusti, Non-Gaussianities in the topological charge distribution of the SU(3) Yang-Mills theory, Phys. Rev. D 92, no. 7, 074502 (2015) [arXiv:1506.06052 [hep-lat]].

[17] C. Bonati, M. D'Elia and A. Scapellato, $\theta$ dependence in SU(3) Yang-Mills theory from analytic continuation, Phys. Rev. D 93, 025028 (2016) [arXiv:1512.01544 [hep-lat]].

[18] C. Bonati, M. D'Elia, P. Rossi and E. Vicari, $\theta$ dependence of $4 D S U(N)$ gauge theories in the large-N limit, Phys. Rev. D 94, 085017 (2016) [arXiv:1607.06360 [hep-lat]].

[19] B. Allés, M. D’Elia, A. Di Giacomo, Topological susceptibility at zero and finite T in SU(3) Yang-Mills theory, Nucl. Phys. B 494, 281 (1997) [Erratum-ibid. B 679, 397 (2004)] arXiv:hep-lat/9605013; Phys. Lett. B 412, 119 (1997) arXiv:hep-lat/9706016; Phys. Lett. B 483, 139 (2000) arXiv:hep-lat/0004020.

[20] C. Gattringer, R. Hoffmann, S. Schaefer, The Topological susceptibility of SU(3) gauge theory near T(c), Phys. Lett. B 535, 358 (2002) arXiv:hep-lat/0203013.

[21] L. Del Debbio, H. Panagopoulos, E. Vicari, Topological susceptibility of $S U(N)$ gauge theories at finite temperature, JHEP 0409, 028 (2004) arXiv:hep-th/0407068.

[22] C. Bonati, M. D'Elia, H. Panagopoulos and E. Vicari, Change of $\theta$ Depen ence in 4D SU(N) Gauge Theories Across the Deconfinement Transition, Phys. Rev. Lett. 110, 25, 252003 (2013) [arXiv:1301.7640 [hep-lat]].

[23] R. Sommer, A New way to set the energy scale in lattice gauge theories and its applications to the static force and alpha-s in SU(2) Yang-Mills theory, Nucl. Phys. B 411, 839 (1994) [hep-lat/9310022].

[24] E. Thomas and A. R. Zhitnitsky, Topological Susceptibility and Contact Term in QCD. A Toy Model, Phys. Rev. D 85, 044039 (2012) [arXiv:1109.2608 [hep-th]].

[25] M. Unsal, Theta dependence, sign problems and topological interference, Phys. Rev. D 86, 105012 (2012), arXiv:1201.6426.

[26] K. Aitken, A. Cherman and M. Ünsal, Vacuum structure of Yang-Mills theory as a function of $\theta$, arXiv:1804.06848 [hep-th]. 